\title{
Considerations for Verticillium Wilt Resistance Evaluation in Potato
}

\author{
K. E. Frost and D. I. Rouse, University of Wisconsin-Madison, Department of Plant Pathology, Madison 53706; \\ and S. H. Jansky, United States Department of Agriculture-Agricultural Research Service and University of Wis- \\ consin-Madison, Department of Horticulture, Madison 53706
}

\begin{abstract}
Frost, K. E., Rouse, D. I., and Jansky, S. H. 2007. Considerations for Verticillium wilt resistance evaluation in potato. Plant Dis. 91:360-367.

Verticillium wilt ( $\mathrm{Vw}$ ), caused by the soilborne fungi Verticillium dahliae and V. albo-atrum, is an important disease of potato (Solanum tuberosum). Host plant resistance is a promising method of Vw control. Culture-based methods that quantify the pathogen in host tissue often are used for $\mathrm{Vw}$ resistance screening. To evaluate the processing time, accuracy, and precision of these methods, 46 clones were planted in a field naturally infested with $V$. dahliae to collect data on visual disease symptoms, pathogen colonization, and yield. In 2002, disease severity explained $43.4 \%$ of the variability of yield loss, but the linear relationship between stem colonization and yield loss was not significant. In 2003, stem colonization explained $57.5 \%$ of the variability of yield loss, whereas disease severity explained $1.7 \%$ of the variability of yield loss. Correlations comparing clone ranks from repeated pathogen measurements indicated that culturing sap from individual stems or bulked stems generated more repeatable clone rankings than culturing dried stems. Clone rankings were more repeatable between years if pathogen measurements were made earlier in the growing season. The results indicate a need to characterize the effect of the environment on the relationship among pathogen population sizes in planta, disease symptoms, and yield loss.
\end{abstract}

Additional keywords: disease resistance

An important disease of potato (Solanum tuberosum L.) is Verticillium wilt $(\mathrm{Vw})$, which causes early plant senescence and yield reductions $(7,32-34)$. $\mathrm{Vw}$ is caused mainly by the soilborne vascular wilt fungi Verticillium dahliae and V. alboatrum (Reinke \& Berthier). However, $V$. dahliae is known to interact synergistically with the parasitic nematode Pratylenchus penetrans to exacerbate the effects of $\mathrm{Vw}$ $(22,23,30)$. Several cultural practices are able to reduce the severity of $\mathrm{Vw}$; however, the amount of control is variable and sometimes low when compared with the economic return associated with chemical control by soil fumigation. Cultural control practices with a positive impact include crop rotation $(6,12,28)$, solarization $(10,19)$, soil amending $(20,37)$, fertility management $(10,11)$, and irrigation management $(4,5,10)$. Biological control has potential, but consistent results have not yet been attained $(13,24,35)$. Soil fumigation is used extensively in many production areas with good success. However, this practice is expensive

Corresponding author: S. H. Jansky

E-mail: shjansky@wisc.edu

Accepted for publication 6 October 2006.

doi:10.1094/PDIS-91-4-0360

This article is in the public domain and not copyrightable. It may be freely reprinted with customary crediting of the source. The American Phytopathological Society, 2007. and requires chemical applicators to follow protocols carefully in order to avoid human health and environmental problems. Several Vw-resistant potato cultivars, such as Red Dale, Ranger Russet, and Defender, have been released, but these have not replaced the most widely grown cultivars. Thus, host plant resistance is a promising method of $\mathrm{Vw}$ control that needs additional development.

Techniques for $\mathrm{Vw}$ resistance screening in potato are abundant in the literature $(9,14,16,29,31,39)$. The easiest technique is to grow plants in a field with a natural infestation of the fungus and look for symptom expression. However, the only characteristic symptoms of the disease are unilateral chlorosis and necrosis in mature plants (17) associated with wilting and a slight brown discoloration of conducting tissue (38). The symptoms are similar to those observed in naturally senescing plants, except that they occur earlier than normal.

To avoid ambiguity associated with visual scoring methods, breeders have developed assays to quantify the amount of stem colonization by $V$. dahliae (Kleb.) in plants grown in naturally infested fields $(8,9,16)$. Correlations between the number of CFU in plant sap or tissue and wilt symptoms $(9,15,16)$ and the number of CFU in plant tissue and yield loss (9) have been reported. More recent studies have reported a poor correlation between stem colonization and symptom expression $(18,21)$.
For a potato breeder, disease assessment provides quantitative data which are used to identify resistant plants. The concept of resistance cannot be uncoupled from the method used to assess the disease or pathogen. Several studies have been conducted to guide researchers in selecting one disease assessment method over another $(26,27,36)$. One study measured the accuracy and precision of disease assays by determining correlations of disease measurements within and among raters (36). In a similar way, simple linear regression also has been used to detect bias among raters of dollar spot disease on Bentgrass (26).

A breeder wants to know whether there is a relationship among repeated assessments of a breeding clone's performance and whether that relationship is genuine. A test-retest method similar to the one proposed by Shokes et. al. (36) can be used to evaluate the relationship among repeated clone assessments and would measure the correlation between clone ranks from repeated pathogen measurements for a set of potato clones using a single method. This correlation is an indication of the overlap of the two rankings, with a high correlation coefficient, close to 1 or -1 , being desired. Similarly, correlation or regression analyses can be used to evaluate the relationship among pathogen count data from different sample dates (26). The correlation coefficients or the slope, y-intercept, and coefficient of determination of the regression could be interpreted as a measure of the precision of a method. Regression analysis also can be used to evaluate the relationship between pathogen population size measurements and some "true" measurement of disease, such as severity or yield loss (3). The parameter values describing this relationship could be interpreted as a measure of a pathogen assay's accuracy for predicting disease.

This study examined some of the advantages and disadvantages of three different Vw resistance screens: root culturing, stem sap culturing from bulked stems and individual stems, and culturing of individual air-dried stems. For each of the $V$. dahliae quantification methods, the active sample process time and the time to results were evaluated. The percentages of zero values obtained and the limits of detection for each method are reported. For each Vw resistance screen, correlation and regression analyses were used to determine the relationship between repeated clone rank- 
ings and repeated pathogen measurements. A method's repeatability was measured by evaluating the relationships among repeated pathogen measurements over time. In addition, method accuracy was measured by evaluating the relationship between colonization scores and both disease severity and yield loss using regression and correlation analyses. Parameter values describing these relationships were used as a measure of a method's accuracy in describing disease severity and yield loss for a set of potato clones. Potential pitfalls were identified for each $V$. dahliae culturing method to select for $\mathrm{Vw}$ resistance. This information is of particular importance to potato breeders, who screen many different clones for $\mathrm{Vw}$ resistance, among other traits, because their screening technique needs to adequately identify $\mathrm{Vw}$ resistance and be both cost and time effective.

\section{MATERIALS AND METHODS}

2002 Field season at Hancock. All fieldwork was performed at the Hancock, WI Agricultural Experiment Station. The V. dahliae-infested field at this location has been planted continuously to potato for more than 30 years and contains approximately $6.5 \mathrm{CFU}$ of $V$. dahliae per gram of soil. On 30 April 2002, 42 tetraploid clones from the Wisconsin Potato Breeding Program, 'Russet Norkotah,' 'Dark Red Norland,' and two highly resistant diploid interspecific hybrids (C287 and C545), were planted in four replicates in each of two trials. In trial 1 , four-hill plots of each clone were planted in each block in the $V$. dahliae screening field and also in a standard potato research field. This trial was used to evaluate disease, plant maturity, plant vigor, and yield. In trial 2, eighthill plots of each clone were planted in each block on the $V$. dahliae screening field. This trial was planted for destructive sampling. Potato plots were maintained using best management practices based on University of Wisconsin-Extension recommendations (1).

Visual ratings. Plots in trial 1 were scored for maturity based on flowering time $(1=$ post-flower, prostrate to $5=$ mostly buds) and vigor $(1=$ low to $5=$ high) on 23 July. The plots were scored for $\mathrm{Vw}$ based on the percent chlorotic, necrotic, or wilted foliage. Vw was scored on 17 July and 2, 16, and 30 August, and the data were used to calculate area under the disease progress curve (AUDPC) for each plot. Additionally, the differences between the mean AUDPC (DAUDPC) of clones in the $V$. dahliae-infested field and those in the standard field were calculated to account for differences in maturity of the various potato clones. The mean AUDPC was used because the locations of the infested and uninfested fields did not allow for a side-by-side comparison of each plot. Additionally, the clone was the experi- mental unit for yield loss assessment. DAUDPC was used as a measure of the annual disease severity.

Field sampling. On 15 and 29 July and 12 and 26 August, two plants of each clone in each block in trial 2 were harvested by hand. A root sample was collected for each of the 46 clones using a bulb planter. Main stems (above the seed tuber) of all plants were collected for all 46 clones.

Pathogen culturing assays. Individual stem sap. For a subset of 20 clones, sap was collected from individual stems. A 10to $15-\mathrm{cm}$ section of every main stem was cut 8 to $10 \mathrm{~cm}$ above the seed tuber. The individual stems were rinsed in tap water, surface disinfested in $1 \% \mathrm{NaOCl}$ for $30 \mathrm{~s}$, rinsed in sterile distilled water, and then wrapped in Parafilm. Each individual main stem was squeezed through a press to extract sap and a 100- $\mu$ l aliquot was plated onto NPX medium (2). Fungal colonies were counted 10 to 14 days after growth at ambient temperature without light using a stereomicroscope.

Dry stem. For the subset of 20 clones, the apical portion (top five nodes) of each main stem in a plot was placed in a paper bag and allowed to air dry for the dry stem culturing assay. Leaves were removed from the dried stem apices and the stems were ground in a Wiley mill with a 40mesh screen. Per sample, $25 \mathrm{mg}$ was plated onto NPX medium. Fungal colonies were allowed to grow for 10 to 14 days at ambient temperature without light. Plant debris then was washed from the plates by gently rubbing the surface of the agar me-

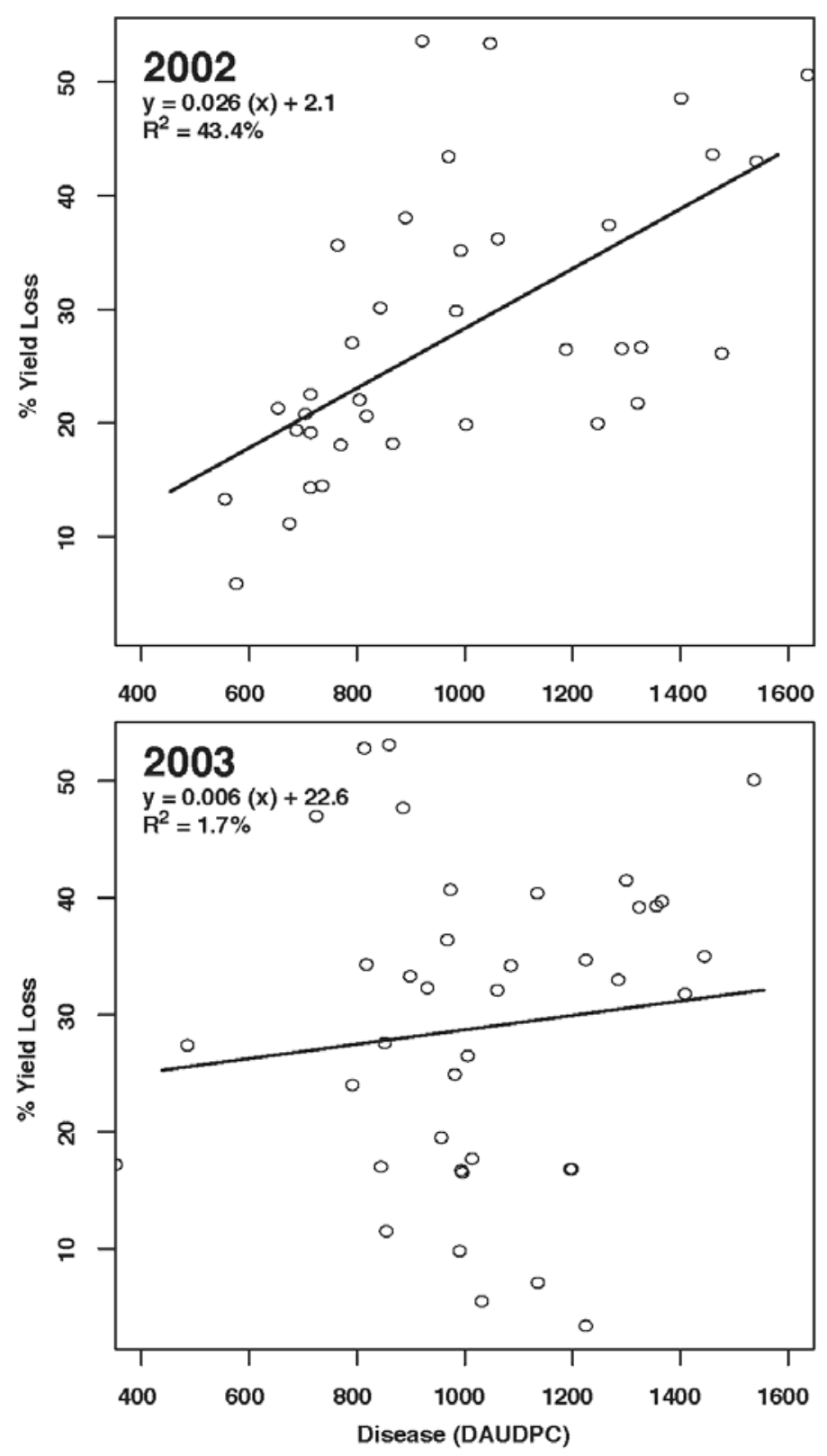

Fig. 1. Accuracy of visual disease rating measured as the ability of visual disease severity ratings (differences between the area under the disease progress curve) to predict percent yield loss in 2002 and 2003. 
dium with fingers under a stream of tap water. Then, fungal colonies on each plate were counted using a stereomicroscope.

Bulked stem sap. For all 46 clones, sap was collected from a bulk sample of all main stems ( 2 to $8 \mathrm{~cm}$ above the seed tuber) of each plant in a plot. The processing procedure for sap was the same for bulked and individual stems.

Root culturing. Root samples of all 46 clones were rinsed in tap water, distilled water, root wash solution (1\% sodium hexametaphosphate and $0.1 \%$ Tergitol NP10), and then sterile distilled water. The main roots were cut into $7-\mathrm{cm}$ sections, seven sections per sample were placed in a petri dish, and NPX medium $\left(50^{\circ} \mathrm{C}\right)$ was poured over them. All plates were incubated for 2 weeks at room temperature without light and $V$. dahliae colonies emerging from the roots were counted using a stereomicroscope.

Yield measurement. All plots were harvested on 4 October and tuber yield was determined. Yield loss was calculated for a clone by the formula: Yield loss $(\%)=100$ $\times[1-($ pooled yield infested plots)/(pooled yield uninfested plots)].

2003 Field season at Hancock. The 2002 trial was repeated in 2003. Seed of four breeding clones from the 2002 trial was not available and substitutions were not made. Seed tubers were planted on 1 May. Vigor and maturity scores were recorded on 21 July. Plots were scored for Vw symptoms on 9, 16, and 23 July and 6 and 19 August. Plots were destructively sampled on 7 and 21 July and 4 and 18 August. In 2003, roots were sampled using a bulb planter on a single date, 1 July. They were washed as previously described; however, in 2003, main roots with lateral feeder roots still attached were placed in petri dishes and NPX medium was poured over them. After the 2-week incubation period, the total length of lateral feeder roots per plate was estimated using the Newman line intersect method (25) and the number of $V$. dahliae colonies was counted.

Data analyses. Accuracy of the various culture-based pathogen assays was determined using regression analyses. For each method, accuracy was assessed by calculating the coefficient of determination $\left(R^{2}\right)$ of the regression of the colonization score on disease severity or yield loss. Regressions were calculated based on the mean performance of each clone at each sample date. Repeatability was determined using mean $V$. dahliae population size regressions between sample dates in each breeding clone. The assessment of repeatability was based on the slope, yintercept, and $R^{2}$.

\section{RESULTS}

Relationships among colonization, disease (DAUDPC), and yield loss. The relationship between visual disease symptoms (DAUDPC) and yield loss varied between the 2002 and 2003 growing seasons (Fig. 1). In 2002, disease assessment explained $43.4 \%$ of the variation in yield loss, and the regression and correlation coefficients were significant. However, in 2003, disease assessment explained less than $2 \%$ of the variation in yield loss and neither the regression nor correlation coefficients were significant.

In 2002, no $V$. dahliae culturing assay was able to explain more than 11 or $17 \%$ of the variation in disease severity or yield loss, respectively, and none of the regression slopes were significantly different from zero $(P<0.05)$ (Table 1 ; Fig. 2$)$. However, in 2003, V. dahliae counts from culturing sap of individual stems and bulked dried stems from 7 July 2003 were able to explain 58 and $31 \%$ of the variation in yield loss, respectively. The regression slopes relating individual stem sap and dried stem $V$. dahliae counts to yield loss also were significantly different from zero $(P<0.05)$ for 7 and 21 July 2003 sample dates. Additionally, regression slopes relating plant sap to disease severity were significantly different from zero, but only explained 11 to $15 \%$ of the variation in disease severity.

Method repeatability. In general, measurements of $V$. dahliae colonization for a set of clones were more repeatable across sample dates when assessed using the sap culturing methods than when dried stem material or roots were cultured (Table
2). No significant correlations between sample dates were found when roots or dried stems were used to assess $V$. dahliae colonization. Sap-culturing measurements of $V$. dahliae colonization were more repeatable, with significant $(P<0.05)$ correlation coefficients ranging from 0.44 to 0.77 , when sample dates were separated by 2 weeks. However, the repeatability decreased when sample dates were separated by 4 weeks, with the exception of the bulked stem sap culturing in 2003, which had a significant correlation $(P<0.05)$ between the first and second $(r=0.69)$ and first and third ( $r=0.328)$ sample dates, but no significant correlation between the second and third sample date. Regression analysis yielded results similar to the correlation analysis. Coefficients of determination for the individual stem sapculturing method ranged from 13 to 59\% and were, on average, higher than the coefficients of determination for the other methods. The slopes of the regression for all methods ranged from 0.23 (plant sap) to 0.89 (stem sap), but were always significantly less than a slope equal to $1.0(P<$ 0.05). Y-intercepts for all methods were always greater than zero, but not always significantly different than zero. Similar patterns in the data were seen when the correlations between the ranks of the clones were calculated (Table 3).

Between years. Between-season repeatability of $V$. dahliae colonization measurement for a set of clones was greater when assessed using the sap-culturing methods than when the assessment was based on the culturing of dried stem material or roots (Table 4). Between seasons, no significant correlations were found when root culturing was used to assess $V$. dahliae colonization. Seven, five, and three out of nine possible correlations were significant for individual stem sap, bulked stem sap, and bulked dry stem culturing, respectively, and absolute values of the correlation coefficients for individual stem sap culturing were, on average, higher than those of the other methods. However, no correlation coefficients were greater than 0.674. Regression analysis yielded results similar to the correlation analysis. Coeffi-

Table 1. Coefficients of determination to evaluate the accuracy of Verticillium dahliae assays in predicting disease measured as the difference between the mean area under the disease progress curve (AUDPC) for a clone or yield loss ${ }^{\mathrm{a}}$

\begin{tabular}{|c|c|c|c|c|c|c|c|c|c|}
\hline \multirow[b]{2}{*}{ Date $^{d}$} & \multicolumn{3}{|c|}{ Stem sap $^{b}$} & \multicolumn{3}{|c|}{ Dried stem $^{b}$} & \multicolumn{3}{|c|}{ Plant sap } \\
\hline & 1 & 2 & 3 & 1 & 2 & 3 & 1 & 2 & 3 \\
\hline \multicolumn{10}{|l|}{2002} \\
\hline DAUDPC & 3.4 & 2.2 & 9.7 & 11.0 & 1.1 & 2.9 & 1.5 & 0.1 & 1.0 \\
\hline Yield loss & 0.1 & 0.5 & 16.5 & 7.4 & $<0.1$ & 10.0 & 2.6 & 1.0 & 0.1 \\
\hline \multicolumn{10}{|l|}{2003} \\
\hline DAUDPC & 19.8 & 13.5 & 0.6 & 3.2 & 2.1 & 1.5 & $15.0 * *$ & $11.2 *$ & $13.0^{*}$ \\
\hline Yield loss & $57.5 * *$ & $45.8 * *$ & 6.5 & $31.3^{*}$ & $28.7 *$ & 8.2 & 2.5 & $17.0 * *$ & 0.3 \\
\hline
\end{tabular}

a Slope of the regression was statistically different than zero at the 0.05 and 0.01 levels (* and $* *$, respectively).

${ }^{\mathrm{b}}$ Sample sizes ranged from 14 to 20.

${ }^{\mathrm{c}}$ Sample sizes ranged from 41 to 46.

${ }^{\mathrm{d}}$ Dates 1, 2, and 3 refer to 15 and 29 July and 12 August 2002 and 7 and 21 July and 4 August 2003, respectively. DAUDPC = differences between the mean AUDPC. 
2002
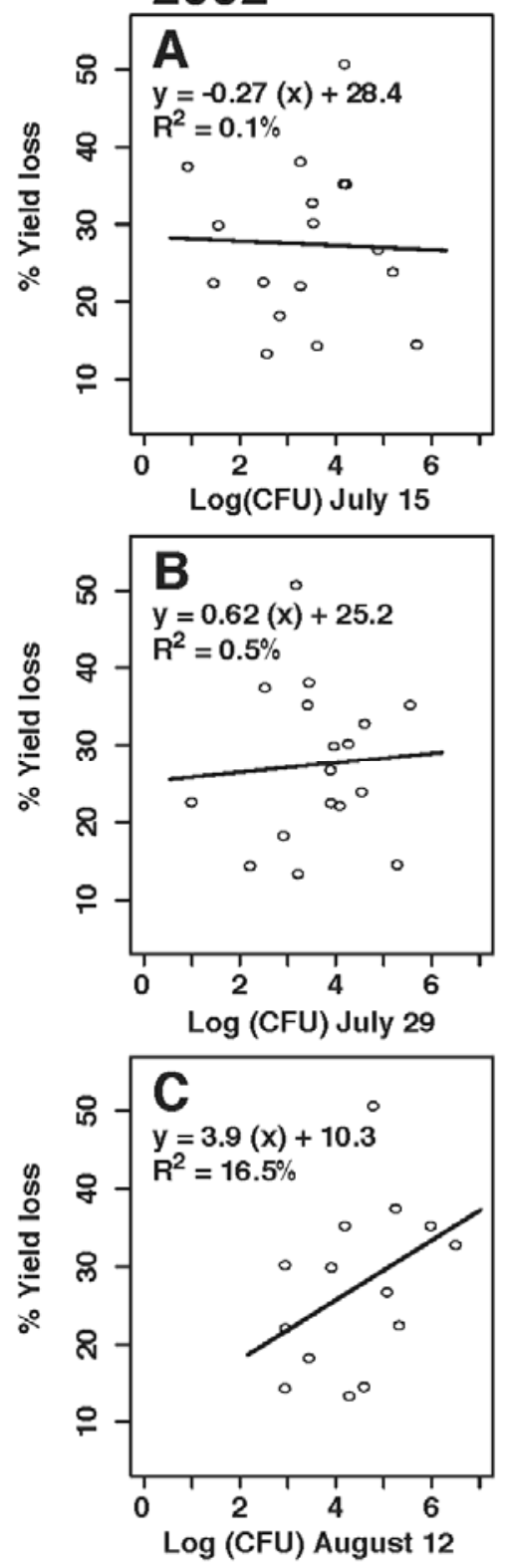

2003
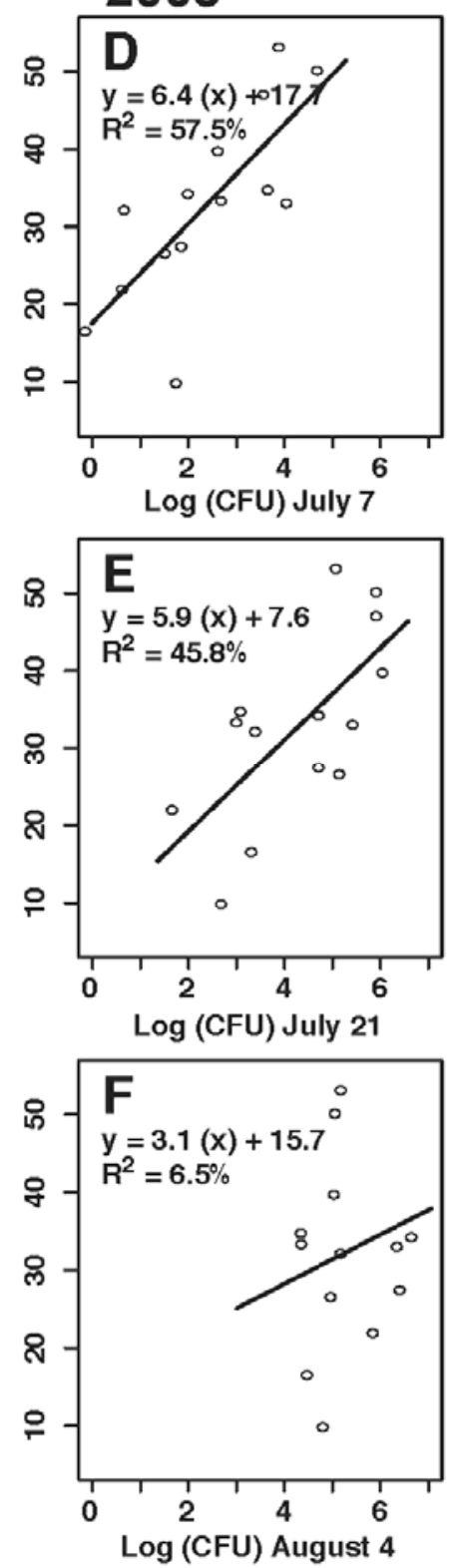

Fig. 2. Accuracy of Verticillium dahliae assays measured as the ability of $V$. dahliae population sizes in potato stem sap to predict percent yield loss on three dates in A-C, 2002 and D-F, 2003.

cients of determination for individual stem sap culturing ranged from 13 to $45 \%$ and were, on average, higher than those for the other methods. Regression slopes for all methods ranged from -0.83 to 1.35 . Five out of nine regression slopes for comparison between years for dried stem culturing were not significantly different from 1.0. However, only 1 of 18 slope values was not significantly different from 1.0 for sapbased V. dahliae assays. In all, 24 of 27 Yintercepts were greater than zero, but not always significantly different than zero. Once again, similar patterns in the data were seen when Spearman's correlation coefficients were calculated (Table 5).

Active process time. The active time to process an experimental unit ranged from 5 to 21 min depending on the method being employed (Table 6). Culturing stem sap was generally less time consuming than plating roots or dried stem material. Enumerating colonies on plates containing dry stem material was more timeconsuming than the other methods because, even after the agar surfaces on the plates were washed, plant debris remained on the plate, making the $V$. dahliae colonies more difficult to distinguish. In addition, it took 4 to 6 weeks longer to obtain results from the culturing of dried stem material because of the length of time required for drying the plant material.

Limit of detection and number of zeros. Within some data sets (one clone at a given sample date), there were culture plates with no $V$. dahliae colonies (Table 7). In both years, the majority of culture plates containing roots did not have $V$. dahliae colonies growing on them. There also was a high frequency of zero values for individual stem sap and dry stem culturing methods. However, for these methods, the data sets never contained $100 \%$ zeros for all four replicates. In general, the percentage of zeros in a data set decreased as the growing season progressed. The limits of detection for each assay were 10 $\mathrm{CFU} / \mathrm{ml}, 40 \mathrm{CFU} / \mathrm{g}$, and $2 \mathrm{CFU} / \mathrm{m}$ for the sap, dried stem, and root culturing meth-

Table 2. Repeatability, measured as the slope, intercept, and coefficient of determination $\left(R^{2}\right)$, of Verticillium dahliae colonization measurement using sap or dried stem culturing methods for a set of clones within a growing season ${ }^{\mathrm{a}}$

\begin{tabular}{|c|c|c|c|c|c|c|c|c|c|}
\hline \multirow[b]{2}{*}{ Year, dates ${ }^{d}$} & \multicolumn{3}{|c|}{ Stem sap $^{b}$} & \multicolumn{3}{|c|}{ Dried stem $^{b}$} & \multicolumn{3}{|c|}{ Plant sap $^{\mathrm{c}}$} \\
\hline & Slope & Intercept & $R^{2}(\%)$ & Slope & Intercept & $R^{2}(\%)$ & Slope & Intercept & $R^{2}(\%)$ \\
\hline \multicolumn{10}{|l|}{2002} \\
\hline $1 / 2$ & $0.69^{*}$ & 1.13 & 47.9 & 0.51 & $2.41 *$ & 14.5 & $0.60 *$ & $3.83 *$ & 26.7 \\
\hline $1 / 3$ & 0.31 & $3.16^{*}$ & 13.0 & $0.81 *$ & 1.40 & 22.7 & $0.29 *$ & $3.78 *$ & 3.8 \\
\hline $2 / 3$ & $0.49 *$ & $2.47^{*}$ & 30.7 & 0.52 & 2.25 & 16.3 & $0.56^{*}$ & 1.51 & 19.4 \\
\hline \multicolumn{10}{|l|}{2003} \\
\hline $1 / 2$ & $0.89 *$ & $1.91 *$ & 49.5 & 0.44 & $3.94 *$ & 7.8 & $0.64 *$ & $2.10^{*}$ & 48.0 \\
\hline $1 / 3$ & 0.56 & $3.74 *$ & 24.1 & 0.48 & $5.77^{*}$ & 8.6 & $0.24 *$ & $1.42 *$ & 10.8 \\
\hline $2 / 3$ & $0.69 *$ & $2.31^{*}$ & 58.6 & 0.38 & $5.02 *$ & 13.7 & 0.23 & $1.29 *$ & 7.9 \\
\hline
\end{tabular}

${ }^{a}$ Root culturing was not included in the table because any given measurement was never able to explain more than $10 \%$ of the variation of a repeated measurement and no slope values were significantly different from $0.0(P<0.05)$. Slope parameters followed by an $*$ are significantly different from zero $(P<$ $0.05)$ and all slopes were significantly different from a value of 1.0 . Y-intercepts followed by an * are significantly different from zero $(P<0.05)$.

${ }^{\mathrm{b}}$ Sample sizes ranged from 16 to 20.

c Sample sizes ranged from 45 to 46 . Plant sap was a bulked sample of all main stems from a plant.

${ }^{\mathrm{d}}$ Comparison of dates within each year. Dates 1, 2, and 3 refer to 15 and 29 July and 12 August 2002 and 7 and 21 July and 4 August 2003 , respectively. 
ods, respectively. These limits might be the cause of some of the zero values.

\section{DISCUSSION}

$V$. dahliae population sizes in planta were not able to explain the variation in either disease severity or yield loss in 2002. Yet, there was a significant relationship between disease severity and yield loss; visual symptoms were able to describe $43 \%$ of the variability in yield loss (Table 1; Figs. 1 and 2). In 2003, Vw symptoms were less severe and were not related to yield loss. However, the mean $V$. dahliae population size for a clone, as measured by sap colonization in individual stems, was able to describe nearly $58 \%$ of the variability in yield loss. In fact, all estimates of $V$. dahliae population sizes in planta were better able to explain the variability in yield loss than was a visual disease assessment. The implication of these data is that selecting for differences in disease resistance in different years or at different times within a year may be difficult if using only quantitative assays of $V$. dahliae population size or disease symptoms.

One might attribute the difference between seasons to a difference in the pathogen population sizes and expect that, because disease symptoms were more severe in 2002, pathogen population sizes would be greater in 2002. However, the pathogen population sizes in a clone were, on average, larger in 2003. For example, the median pathogen population size on 29 July 2002, was 33 whereas the median $V$. dahliae population size on 21 July 2003 was 80. In 2003, the presence of $V$. dahliae in potato stems resembled that of an endophyte rather than a pathogen if one were to consider only disease symptoms and not yield loss.

Potato growers often make comments about a particular year being an "early dying year." The year 2002 was considered to be an early dying year and 2003 was one of the best potato production years in Wisconsin history. The 2002 growing season was vastly different from the 2003 growing season. For example, the average

Table 3. Repeatability, measured as the Spearman's (rank) correlation coefficient ( $r$ ) of Verticillium dahliae colonization measurement using sap, dried stem, or root culturing methods for a set of clones across sample dates within a growing season ${ }^{\mathrm{a}}$

\begin{tabular}{ccccc}
\hline & \multicolumn{4}{c}{$r$} \\
\cline { 2 - 5 } Year, date $^{\mathbf{b}}$ & Stem sap $^{\mathbf{c}}$ & Dried stem $^{\mathbf{c}}$ & Root colonization $^{\mathbf{d}}$ & Plant sap $^{\mathbf{d}, \mathbf{e}}$ \\
\hline 2002 & & & & \\
$1-2$ & $0.644^{* *}$ & 0.157 & 0.069 & $0.486^{* *}$ \\
$1-3$ & 0.259 & 0.316 & $\ldots$ & 0.189 \\
$1-4$ & $\ldots$ & $\ldots$ & -0.178 & $\ldots$ \\
$2-3$ & 0.405 & 0.347 & $\ldots$ & $\ldots .328^{*}$ \\
$2-4$ & $\ldots$ & $\ldots$ & -0.060 & $\ldots$ \\
2003 & & & $\ldots$ & $0.611^{* *}$ \\
$1-2$ & $0.649^{* *}$ & 0.106 & $\ldots$ & 0.266 \\
$1-3$ & 0.381 & 0.047 & $\ldots$ & 0.224 \\
$2-3$ & $0.591^{*}$ & 0.074 & $\ldots$ & $\ldots$ \\
\hline
\end{tabular}

a $\ldots=$ Stems were not sampled on 26 August 2002 and roots were sampled on only a single date in $2003 ; *$ and $* *=$ significant at the 0.05 and 0.01 probability level, respectively.

${ }^{\mathrm{b}}$ Comparison of dates within each year. Dates 1, 2, 3, and 4 refer to 15 and 29 July and 12 and 26 August 2002, respectively. Dates 1, 2, and 3 refer to 7 and 21 July and 4 August 2003, respectively.

${ }^{\mathrm{c}}$ Sample sizes ranged from 16 to 20.

${ }^{\mathrm{d}}$ Sample sizes ranged from 45 to 46.

e Plant sap was a bulked sample of all main stems from a plant. temperature (7 May to 31 July) for 2002 and 2003 were 28 and $27^{\circ} \mathrm{C}$, respectively. However, the variability of temperature in 2002 was much higher (variation of daily maximum temperature $=35$ and variation of daily minimum temperature $=28$ ) than that in 2003 (maximum $=22$ and minimum $=14$ ). Additionally, the cumulative rainfall and irrigation for the 2002 and 2003 growing seasons were different (67.1 and 40.8 $\mathrm{cm})$ and the rain came in very different ways. In 2003, the rain or irrigation averaged $0.83 \mathrm{~cm}$ (variation of rain events = 0.050 ) per event and, in 2002, rainfall averaged $1.46 \mathrm{~cm}$ (variation of rain events $=0.69$ ) per event. However, in 2002, a single rain event dropped nearly $21 \mathrm{~cm}$ and may have affected $\mathrm{VW}_{\mathrm{w}}$ progression by leaching nitrogen from the soil. Although the weather data are anecdotal, we feel that environmental variation plays a large role in the seasonal variability of $\mathrm{Vw}$ symptom severity. Therefore, it is necessary to try to determine the relative importance of environment on the host, pathogen population sizes, and subsequent development of disease.

Plant breeders evaluate large numbers of clones each year, despite time and monetary constraints. Therefore, it becomes important to know whether the set of clones selected for $\mathrm{VW}_{\mathrm{w}}$ resistance at the end of the growing season would be the same as those selected if sampling occurred earlier in the season or if the sampling occurred in a different season. Regression analysis was used to examine the relationship between repeated pathogen population size measurements. For this analysis, methods that had the highest repeatability would be those in which the regression slopes are closest to one and the $y$-intercepts are closest to zero for comparisons between two measures of resistance at two different times. Deviation of a slope from a value of one would indicate the presence of a systemic bias, and yintercepts deviating from zero would indicate a constant bias in a method (26). In

Table 4. Repeatability, measured as the slope, intercept, and coefficient of determination $\left(R^{2}\right)$, of Verticillium dahliae colonization measurement using sap or dried stem culturing methods for a set of clones between growing seasons ${ }^{\mathrm{a}}$

\begin{tabular}{|c|c|c|c|c|c|c|c|c|c|}
\hline \multirow[b]{2}{*}{$2002 / 2003^{d}$} & \multicolumn{3}{|c|}{ Stem sap ${ }^{b}$} & \multicolumn{3}{|c|}{ Dried stem $^{b}$} & \multicolumn{3}{|c|}{ Plant sap ${ }^{c}$} \\
\hline & Slope & Intercept & $R^{2}(\%)$ & Slope & Intercept & $R^{2}(\%)$ & Slope & Intercept & $R^{2}(\%)$ \\
\hline $1 / 1$ & $0.58 *$ & 0.32 & 33.5 & 0.56 & 0.26 & 12.9 & $0.41 *$ & $2.33 *$ & 11.2 \\
\hline $1 / 2$ & $0.83^{*}$ & 1.26 & 44.9 & $1.35 * *$ & -0.23 & 29.8 & $0.57 *$ & $2.68 *$ & 28.3 \\
\hline $1 / 3$ & $0.56^{*}$ & $3.20 *$ & 25.6 & $0.97^{1}$ & 3.18 & 14.2 & $0.48 *$ & $0.82 *$ & 28.3 \\
\hline $2 / 1$ & $0.63 *$ & -0.04 & 34.7 & 0.13 & 1.85 & 1.3 & 0.31 & 1.77 & 8.1 \\
\hline $2 / 2$ & $0.85^{*}$ & 0.90 & 42.2 & $0.96^{* *} *$ & 0.57 & 27.2 & $0.60 *$ & 0.95 & 39.2 \\
\hline $2 / 3$ & $0.76^{*}$ & $2.35^{*}$ & 41.2 & $0.86^{1}$ & 3.00 & 19.8 & $0.30 *$ & 0.56 & 13.3 \\
\hline $3 / 1$ & 0.28 & 0.76 & 6.9 & 0.05 & 2.23 & 0.3 & -0.10 & $4.01 *$ & 1.6 \\
\hline $3 / 2$ & $1.02 * *$ & -0.83 & 45.5 & $0.92 * *$ & 0.61 & 42.0 & -0.03 & $4.49 *$ & 0.1 \\
\hline $3 / 3$ & 0.51 & 2.58 & 13.9 & 0.47 & $4.66^{*}$ & 10.5 & 0.09 & $1.83 *$ & 2.1 \\
\hline
\end{tabular}

a Slopes followed by $*$ were significantly $(P<0.05)$ different from zero and those followed by $* *$ were not significantly $(P<0.05)$ different from one. Yintercepts followed by * were significantly $(P<0.05)$ different from zero.

b Sample sizes ranged from 13 to 16 .

c Sample size was 42 .

${ }^{\mathrm{d}}$ Comparison of sampling dates between years 2002 and 2003. Dates 1, 2, and 3 refer to 15 and 29 July and 12 August 2002 and 7 and 21 July and 4 August 2003, respectively. 
this study, both systemic and constant biases were found in all of the pathogenquantifying methods that were evaluated. Y-intercepts were always greater than zero, indicating that pathogen population sizes, as measured by the various methods, were higher later in the growing season. Within a single year, regression slopes were always significantly less than one. A general conclusion from the regression slope data is difficult to construct without further analysis. However, deviation from a slope of one indicates that pathogen population size dynamics differ among clones. These dynamics will be discussed elsewhere (unpublished data). Although clone performance was variable across seasons, large variations in clone ranks were not seen. This is similar to what we have seen when using these methods to assess $\mathrm{Vw}$ resistance in other breeding materials. It might be expected that the relationship between repeated clone rankings would be similar to that seen in the pathogen measurements. That was the case in this study. Spearman's correlation coefficients ranged from -0.178 to 0.649 , but were never close to 1 (or -1$)$.

These results reveal that it is important for a breeder to consider the timing of an assay within the growing season. There appear to be seasonal dynamics of $V$. dahliae population sizes that differ among potato clones and the dynamics might not be entirely controlled by host genetics. In particular, our results indicate that assay- ing pathogen population sizes in planta earlier in the growing season might better predict percent yield loss than assaying pathogen population sizes later in the growing season.

Many other factors may lead to variation in results when enumerating the pathogenic fungal populations from stems. One is worth mentioning here to emphasize that classification of resistance to $V$. dahliae may depend on the analytical method used to assess disease. This study used three different culturing methods to evaluate resistance to $V$. dahliae. However, the CFU was not identified on the culture plates. The $V$. dahliae colonies on an individual plate could have grown from mycelial fragments, conidia, or microsclerotia, and each fungal propagule could relate to disease (and disease resistance) in a different way. Not knowing the vegetative propagule leaves two important questions unanswered. (i) Does a single type of $V$. dahliae resistance screen identify the same resistance mechanism in different potato clones? (ii) Do the different $V$. dahliae resistance screens identify the same resistance mechanism in different potato clones? Additional research is needed on the physiological basis of Vw resistance.

The amount of time it takes to get results for an assay is an important consideration for all plant breeders because it relates, sometimes directly, to the number of clones that can be evaluated. The exception, of course, is when the variability of

Table 5. Repeatability, measured as the Spearman's (rank) correlation coefficient ( $r$ ) of Verticillium dahliae colonization measurement using sap or dried stem culturing methods for a set of clones between growing seasons ${ }^{\mathrm{a}}$

\begin{tabular}{lcccc}
\hline $\mathbf{2 0 0 2}^{\mathbf{b}}$ & $\mathbf{2 0 0 3}^{\mathbf{b}}$ & Stem sap $^{\mathbf{c}}$ & Dried stem $^{\mathbf{c}}$ & Plant sap $^{\mathbf{d}}$ \\
\hline 1 & 1 & $0.568^{*}$ & 0.291 & $0.310^{*}$ \\
1 & 2 & $0.619^{*}$ & $0.615^{*}$ & $0.494^{* *}$ \\
1 & 3 & 0.373 & 0.497 & $0.505^{* *}$ \\
2 & 1 & $0.547^{*}$ & -0.047 & 0.121 \\
2 & 2 & $0.535^{*}$ & $0.625^{*}$ & $0.421^{* *}$ \\
2 & 3 & $0.615^{*}$ & $0.512^{*}$ & 0.269 \\
3 & 1 & 0.319 & -0.126 & -0.197 \\
3 & 2 & $0.694^{*}$ & 0.397 & -0.228 \\
3 & 3 & 0.295 & 0.476 & 0.125 \\
\hline
\end{tabular}

a $\ldots=$ Stems were not sampled on 26 August 2002 and roots were sampled on only a single date in $2003 ; *$ and $* *=$ significant at the 0.05 and 0.01 probability level, respectively.

${ }^{\mathrm{b}}$ Sampling dates within each year. Dates 1, 2, and 3 refer to 15 and 29 July and 12 August 2002 and 7 and 21 July and 4 August 2003, respectively.

${ }^{c}$ Sample sizes ranged from 13 to 16.

d Sample size was 42. the information gained is high, necessitating an increased sample size. For example, an assay which consumes a relatively small amount of time might not be desirable if the information gained is variable. Other time considerations also exist. In this study, the amount of time it took to obtain results from dry stem culturing was considerably longer than sap or root culturing because of the drying time for stem material. However, the relative stability of dried stems compared with plant sap offers the flexibility to choose when culture plates are made.

Many plates contained no $V$. dahliae colonies. These zero values are a problem, because the information they convey carries a mixed signal. The plant can either be (i) not infected or (ii) infected but with the pathogen remaining below the assay's limit of detection. Biochemical techniques have the ability to decrease the limit of detection such that one could adequately distinguish between a plant that is infected and one that is uninfected. In our preliminary evaluations, we have found that polymerase chain reaction (PCR)-based $V$. dahliae detection and culture plating give the same positive or negative result nearly $79 \%$ of the time and, $9 \%$ of the time, PCR detected $V$. dahliae in stem sap when no pathogen could be cultured. The limit of detection for sap culturing was higher than that for PCR analysis. However, $12 \%$ of the time, $V$. dahliae grew from cultured sap but was not detected by PCR. Perhaps some colonies that were counted as $V$. dahliae were actually a different species of Verticillium. One concern with PCR detection, however, is that it cannot determine whether a pathogen is alive or dead.

A potato breeder encounters many difficulties when trying to identify $\mathrm{Vw}$ resistance in early generation materials. For one, numbers of seed pieces for each clone are low, making the accurate assessment of yield loss difficult. There is also a drastic increase in the time investment when one moves from using visual disease screening methods to a culture-based pathogenscreening method. Our data indicate that, in central Wisconsin, culturing sap from individual stems can provide more accurate and repeatable estimates of $\mathrm{Vw}$ resistance on a set of potato clones in a shorter period of time than can individual dried-

Table 6. Amount of active process time and the amount of time until results are obtained for root, stem sap, and dried stem culturing methods

\begin{tabular}{lccc}
\hline Activity & Root (time/plant) & Sap plating (time/stem) & Stem dry (time/stem) \\
\hline Collecting samples from the field (min) & 1 & 1 & 1 \\
Sample preparation, live tissue (min) $^{\mathrm{a}}$ & 8 to 15 & 3 to 4 & $\ldots$ \\
Sample preparation, dry tissue (min) & $\mathrm{b}$ & $\ldots$ & 10 to 15 \\
Enumerating fungus (min) & 1 to 4 & 1 to 4 & 1 to 5 \\
Active process time (min) & 10 to 20 & 0 & 12 to 21 \\
Drying time (days) & 0 & 10 to 14 & 28 to 42 \\
Incubation (days) & 10 to 14 & $>10$ to 14 & 10 to 14 \\
Time to results (days) & $>10$ to 14 & $>38$ to 56 \\
\hline
\end{tabular}

\footnotetext{
${ }^{\text {a }}$ For example, surface disinfesting and plating.
}

${ }^{\mathrm{b}}$ For example, grinding, weighing, and plating. 
Table 7. Range in the percentage of zeros at a sample date among Verticillium dahliae clone data sets, except clones C545 and C287

\begin{tabular}{lcccc}
\hline & \multicolumn{4}{c}{ Range per sample date $(\boldsymbol{\%})^{\mathbf{a}}$} \\
\cline { 2 - 5 } Year, method & $\mathbf{1}$ & $\mathbf{2}$ & $\mathbf{3}$ & $\mathbf{4}$ \\
\hline 2002 & & & & \\
Root colonization & $\mathrm{b}$ & $25-100$ & $\ldots$ & $33-100$ \\
Stem colonization sap $^{\mathrm{c}}$ & $0-50$ & $0-46$ & $0-26$ & $\ldots$ \\
Plant colonization sap $^{\mathrm{b}}$ & $13-86$ & $0-100$ & $0-86$ & $\ldots$ \\
Stem colonization dry $^{\mathrm{c}}$ & $12-60$ & $7-61$ & $16-85$ & $\ldots$ \\
2003 & & & & $\ldots$ \\
Root colonization $^{\mathrm{b}}$ & $88-100$ & $\ldots$ & $0-13$ & $\ldots$ \\
Stem colonization sap $^{\mathrm{c}}$ & $14-61$ & $0-100$ & $0-100$ & $0-100$ \\
Plant colonization sap $^{\mathrm{b}}$ & $0-100$ & $0-57$ & $0-13$ & $\ldots$ \\
Stem colonization dry $^{\mathrm{c}}$ & $28-78$ & 0 & \\
\hline
\end{tabular}

${ }^{a}$ Dates 1, 2, 3, and 4 refer to 15 and 29 July and 12 and 26 August 2002, respectively. Dates 1, 2, 3, and 4 refer to 7 and 21 July and 4 and 18 August 2003, respectively. Fungal contamination was a problem for roots plated on 12 August $2002 ; \ldots=$ no data collected

b Data sets consisted of 6 to 8 plates within a clone.

${ }^{\mathrm{c}}$ Data sets consisted of 4 to 30 plates within a clone.

stem culturing or culturing sap from bulk stems of a plant. However, the relationship between $V$. dahliae population size estimates using culture-based methods and either disease severity or yield loss is seasonally variable (Figs. 1 and 2) and may provide useful information only if environmental conditions are appropriate. The data presented here illustrate that there is a need to study the effect of the environment on host plant resistance and the pathogen population sizes resulting in disease symptoms or yield loss. In addition, there may be a need to tailor the screening method to the type of resistance found in the germ plasm pool of a breeding program. For example, pathogen population size data from sap may indicate the ability of the fungus to proliferate in living tissue, whereas dried stem data may reflect the ability of the pathogen to respond to plant senescence. In the future, it might be beneficial for pathologists and breeders across regions to find practical screening methods that meet the specific needs of breeding programs and apply reliability tests to those techniques in the different locations. This will help to determine the best method for a given location and situation, and may help researchers to better interpret data for the same clone in different regions.

\section{ACKNOWLEDGMENTS}

This work was funded in part by the University of Wisconsin Consortium for Extension and Research in Agriculture and Natural Resources.

\section{LITERATURE CITED}

1. Binning, L. K., Boerboom, C. M., Bundy, L. G., Delahaut, K. A., Harrison, H. C., Kelling, K. A., Mahr, D. L., Mahr, S. E. R., Michaelis, B. A., Stevenson, W. R., Wedberg, J. L., and Wyman, J. A. 2002. Commercial Vegetable Production in Wisconsin. Cooperative Extension Publishing, Madison, WI.

2. Butterfield, E. J., and DeVay, J. E. 1977. Reassessment of soil assays for Verticillium dahliae. Phytopathology (67):1073-1078.

3. Campbell, C. L., and Madden, L. V. 1990. Introduction to Plant Disease Epidemiology. John Wiley \& Sons, Inc., New York.

4. Cappaert, M. R., Powelson, M. L., Christen- sen, N. W., and Crowe, F. J. 1992. Influence of irrigation on severity of potato early dying and tuber yield. Phytopathology 82 (12):14481453.

5. Cappaert, M. R., Powelson, M. L., Christensen, N. W., Stevenson, W. R., and Rouse, D. I. 1994. Assessment of irrigation as a method of managing potato early dying. Phytopathology 84 (12):792-800.

6. Chen, J., Bird, G. W., and Mather, R. L. 1995. Impact of multi-year cropping regimes on Solanum tuberosum tuber yields in the presence of Pratylenchus penetrans and Verticillium dahliae. J. Nematol. 27 (4 Suppl.):654-660.

7. Davis, J. R. 1981. Verticillium wilt of potato in southeastern Idaho. University of Idaho Curr. Inf. Ser. No. 564

8. Davis, J. R., Huisman, O. C., Everson, D. O., and Schneider, A. T. 2001. Verticillium wilt of potato: a model of key factors related to disease severity and tuber yield in southeastern Idaho. Am. J. Potato Res. 78:291-300.

9. Davis, J. R., Pavek, J. J., and Corsini, D. L. 1983. A sensitive method for quantifying Verticillium dahliae colonization in plant tissue and evaluating resistance among potato genotypes. Phytopathology 73:1009-1014.

10. Davis, J. R., and Sorensen, L. H. 1986. Influence of soil solarization at moderate temperatures on potato Solanum tuberosum genotypes with differing resistance to Verticillium dahliae. Phytopathology 76 (10):1021-1026.

11. Davis, J. R., Sorensen, L. H., Stark, J. C., and Westermann, D. T. 1990. Fertility and management practices to control Verticillium wilt of the Russet Burbank potato. Am. Potato J. (1):55-66.

12. Easton, G. D., Nagle, M. E., and Seymour, M. D. 1992. Potato production and incidence of Verticillium dahliae following rotation to nonhost crops and soil fumigation in the state of Washington. Am. Potato J. 69 (8):489-502.

13. Entry, J. A., Strasbaugh, C. A., and Sojka, R. E. 2000. Wood chip-polyacrylamide medium for biocontrol bacteria decreases Verticillium dahliae infection on potato. Biocontrol Sci. Technol. 10:677-686.

14. Heinz, R. A., and Platt, H. W. 2000. Improved DNA extraction method for Verticillium detection and quantification in large-scale studies using PCR based techniques. Can. J. Plant Pathol. 22:117-121.

15. Hoyos, G. P., Lauer, F. I., and Anderson, N. A. 1993. Early detection of Verticillium wilt resistance in a potato breeding program. Am. Potato J. 70:535-541.

16. Hoyos, G. P., Zambino, P. J., and Anderson, N. A. 1991. An assay to quantify vascular coloni- zation of potato by Verticillium dahliae. Am. Potato J. 68:727-742.

17. Issac, I., and Harrison, J. A .C. 1968. The symptoms and causal agents of early-dying disease (Verticillium wilt) of potatoes. Ann. Appl. Biol. 61:231-244.

18. Jansky, S. H., and Rouse, D. I. 2000. Identification of potato interspecific hybrids resistant to Verticillium wilt and determination of criteria for resistance assessment. Potato Res. 43:413-426.

19. Lazarovits, G., Hawke, M. A., Tomlin, A. D Olthof, T. H. A., and Squire, S. 1991. Soil solarization to control Verticillium dahliae and Pratylenchus penetrans on potatoes in central Ontario Canada. Can. J. Plant Pathol. 13 (2):116-123.

20. Lazarovits, G., Tenuta, M., and Conn, K. L. 2001. Organic amendments as a disease control strategy for soilborne diseases of highvalue agricultural crops. Australas. Plant Pathol. 30 (2):111-117.

21. Lynch, D. R., Kawchuk, L. M., Hachey, J., Bains, P. S., and Howard, R. J. 1997. Identification of a gene conferring high levels of resistance to Verticillium wilt in Solanum chacoense. Plant Dis. 81:1011-1014.

22. MacGuidwin, A. E., and Rouse, D. I. 1990. Role of Pratylenchus penetrans in the potato early dying disease of Russet Burbank potato. Phytopathology 80:1077-1082.

23. Martin, M. J., Riedel, R. M., and Rowe, R. C. 1982. Verticillium dahliae and Pratylenchus penetrans: Interactions in the early dying complex of potato in Ohio. Phytopathology 72:640-644.

24. Nagtzaam, M. P. M., Bollen, G. J., and Termorshuizen, A. J. 1998. Efficacy of Talaromyces flavus alone or in combination with other antagonists in controlling Verticillium dahliae in growth chamber experiments. J. Phytopathol. (Berlin) 146:165-173.

25. Newman, E. I. 1966. A method of estimating the total length of root in a sample. J. Appl. Ecol. 3:139-145.

26. Nutter, F. W., Jr., Gleason, M. L., Jenco, J. H., and Christians, N. C. 1993. Assessing the accuracy, intra-rater repeatability, and inter-rater reliability of disease assessment systems. Phytopathology $83(8): 806-812$.

27. O'Brien, R. Douglas, and van Bruggen, A. H. C. 1992. Accuracy, precision, and correlation to yield loss of disease severity scales for corky root of lettuce. Phytopathology 82 (1):91-96.

28. O'Sullivan, J. 1978. Effects of rotation and nitrogen on yield and quality of potatoes. Can. J. Plant Sci. 58 (2):475-484.

29. Plasencia, J., Jemmerson, R., and Banttari, E. E. 1996. Production and characterization of monoclonal antibodies to Verticillium dahliae and development of a quantitative immunoassay for fungal biomass. Phytopathology 86:170-176.

30. Powelson, M. L., and Rowe, R. C. 1993. Biology and management of early dying of potatoes. Annu. Rev. Phytopathol. 31:111-126.

31. Robb, J., Hu, X., Platt, H., and Naza, R. 1994. PCR-based assays for the detection and quantification of Verticillium species in potato. In: Modern Assays for Plant Pathogenic Fungi. A. Schots, F. M. Dewey, and R. P. Oliver, eds. CAB International, Wallingford, UK.

32. Rouse, D. I. 1985. Some approaches to prediction of potato early dying disease severity. Am. Potato J. 62:187-193.

33. Rowe, R. C. 1985. Potato early dying: a serious threat to the potato industry. Am. Potato J. 62:157-161.

34. Rowe, R. C., Davis, J. R., Powelson, M. L., and Rouse, D. I. 1987. Potato early dying: causal agents and management strategies. Plant Dis. 71:482-489.

35. Sessitsch, A., Reiter, B., and Berg, G. 2004. 
Endophytic bacterial communities of fieldgrown potato plants and their plant-growthpromoting and antagonistic abilities. Can. J. Microbiol. 50:239-249.

36. Shokes, F. M., Berger, R. D., Smith, D. H., and Rasp, J. M. 1987. Reliability of disease assessment procedures: A case study with late leafspot of peanut. Oleagineux 42 (6):245-251.

37. Soltani, N., Conn, K. L., Abbasi, P. A., and Lazarovits, G. 2002. Reduction of potato scab and Verticillium wilt with ammonium lignosulfonate soil amendment in four Ontario potato fields. Can. J. Plant Pathol. 24 (3):332-339.

38. Stevenson, W. 2001. Compendium of Potato
Diseases. American Phytopathological Society Press, St. Paul, MN

39. Sundaram, S., Plasencia, J., and Bantarri, E. E. 1991. Enzyme-linked immunosorbent assay for detection of Verticillium spp. using antisera produced to $V$. dahliae from potato. Phytopathology 81:1485-1489. 